\title{
IMMEDIATE COMMUNICATION Methamphetamine abuse impairs motor cortical plasticity and function
}

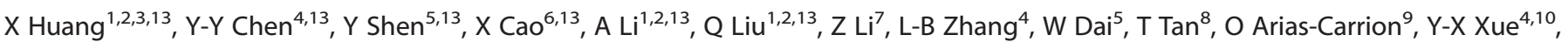 \\ $\mathrm{H} \mathrm{Su}^{11}$ and T-F Yuan ${ }^{1,2,3,12}$
}

Exposure to addictive drugs triggers synaptic plasticity in reward-related brain regions, such as the midbrain, nucleus accumbens and the prefrontal cortex. Effects of chronic drug exposure on other brain areas have not been fully investigated. Here, we characterize synaptic plasticity in motor cortex after methamphetamine self-administration in rats. We show that this causes a loss of corticostriatal plasticity in rat brain slices and impaired motor learning in the rotarod task. These findings are paralleled by the observation of a lack of transcranial magnetic stimulation-induced potentiation or depression of motor evoked potentials in human patients with addiction, along with poor performance in rotary pursuit task. Taken together, our results suggest that chronic methamphetamine use can affect behavioral performance via drug-evoked synaptic plasticity occluding physiological motor learning.

Molecular Psychiatry (2017) 22, 1274-1281; doi:10.1038/mp.2017.143; published online 25 July 2017

\section{INTRODUCTION}

Drug-evoked synaptic plasticity has been proposed as a neural substrate of adaptive behavior. ${ }^{1,2}$ Initial exposure to an addictive drug leads to insertion of calcium-permeable AMPA (a-amino-3hydroxy-5-methyl-4-isoxazolepropionic acid) receptors and calcium-impermeable $\mathrm{N}$-methyl-D-aspartate (NMDA) receptors in dopamine (DA) neurons of the ventral tegmental area; ${ }^{3,4}$ chronic drug exposure also alters synaptic transmission in medium spiny neurons of the nucleus accumbens synapses and, in some cases, also at cortical synapses., 5

Most of these studies focused on drug-evoked synaptic plasticity in subcortical regions (for example, ventral striatum, amygdala, ventral tegmental area) and prefrontal cortex, ${ }_{1}^{7,8}$ all of which receive projections from midbrain DA neurons. Sensory and motor areas receive only sparse DA inputs and have not been extensively studied. ${ }^{9}$ However, evidence for drug-adaptive function also exists for these cortical regions. For instance, brain imaging studies in humans with cocaine or nicotine reveal a cueinduced activation of the sensory association cortex and motor cortex that reflects craving status and predicts relapse of the addicted patients. ${ }^{10-13}$ Chronic exposure to methamphetamine causes structural deficits and altered DA receptor function in the cortical-striatal projections in humans. ${ }^{14}$ Animal studies further reported a cocaine-altered expression in genes of the dorsal striatum associated with motor learning. ${ }^{15}$ Finally, drug abuse accompanies compulsive behavior and drug seeking that could result from altered motor-striatal plasticity and functioning.

In the present study we characterized synaptic plasticity at the motor cortex to the dorsal striatum in rats self-administrated methamphetamine. We then combined transcranial magnetic stimulation (TMS) to monitor plasticity of the motor evoked potential (MEP) in humans to probe a potential plasticity deficit in methamphetamine abusers.

\section{MATERIALS AND METHODS}

Animal subjects

A total of 23 male Sprague-Dawley rats (260-300 g, Beijing University Animal Center, Beijing, China) were subjected for methamphetamine self-administration (SA) training as previously described $^{16,17}$ and 16 male rats were employed as the control (random assignment, animal sample size not preestimated). All animal experimental procedures have been approved by the ethic committee of animal research at Nanjing Normal University, Shanghai Jiaotong University and Beijing University.

For self-administration, the training chambers (AniLab Software \& Instruments, Ningbo, Zhejiang, China) were equipped with two nose-poke detectors (AniLab Software \& Instruments) located $5 \mathrm{~cm}$ above the floor of the chambers. Nose pokes in one (active) opening led to methamphetamine infusions that were accompanied by a $5 \mathrm{~s}$ tone-light cue. Nose pokes in the other (inactive)

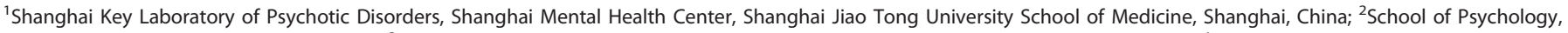

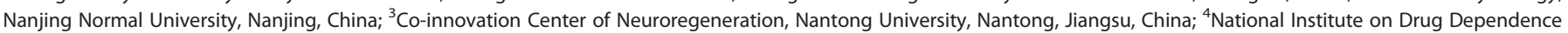

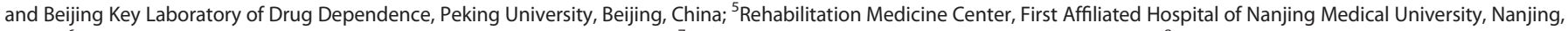

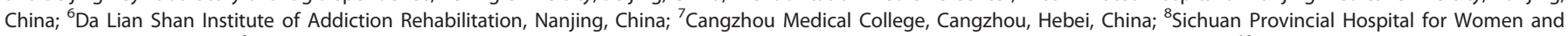

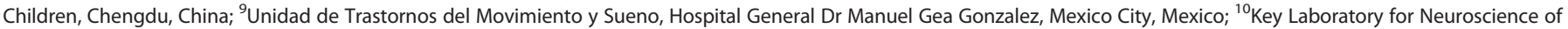

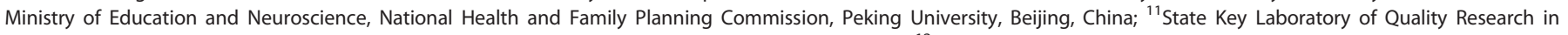

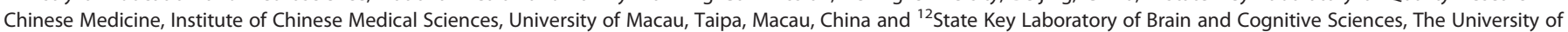

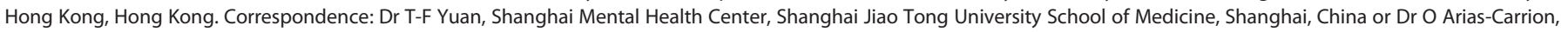

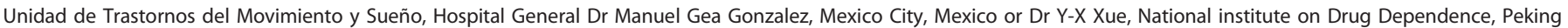
University, Beijing, China or Dr H Su, University of Macau, Macau, China.

E-mail: ytf0707@126.com or arias@ciencias.unam.mx or yanxuexue@bjmu.edu.cn or huanxingsu@umac.mo

${ }^{13}$ These authors share first authorship.

Received 15 December 2016; revised 8 May 2017; accepted 9 May 2017; published online 25 July 2017 
were also recorded but had no consequence. In the present study, all animals were included for analyses (not in blind manner).

\section{Human subjects}

A total of 56 male addicts (methamphetamine only (METH) group, treatment seeking) (26-55 years old, right handed) were recruited for the present study and 35 healthy male subjects as controls (24-54 years old, right handed). There were no differences in age, smoking status or alcohol intake history between the two groups. All subjects reported no use of drugs in the 2 weeks before the investigation (abstinence from drug use ranged from 2 weeks to 3 months) and this was verified by urine sample examination; all subjects reported intact sleep before the experiment.

All the subjects recruited for the study participated voluntarily, and signed the written informed consent forms. Subjects reported no prior history of head trauma, epilepsy, heart diseases or metal implants in the body. The study has been approved by the ethics committee of Shanghai Mental Health Center and Nanjing Normal University, and all experimental procedures followed the guidelines of human medical research (Declaration of Helsinki). The clinical trial registration was ChiCTR-IOR-16008060 (Cortical plasticity of addicts) at http://www.chictr.org.cn.

\section{Criteria for METH abuse}

The abuse of METH was defined by the following criteria: (1) only METH use (not used with other drugs, for example, heroin) for at least 2 years; (2) METH intake of minimum 3 times per week, for a period at least a month (many METH users change their intake frequency and those with occasional drug use were not included); (3) dosage of $>0.3 \mathrm{~g}$ per day (range $0.3-2 \mathrm{~g}$ ); and (4) did not receive additional drug therapy to quit addiction.

\section{Brain slice electrophysiology}

For brain slice preparation, rats were deeply anesthetized with isoflurane and decapitated. Brains were quickly removed and submerged in cutting solution (in mM as follows: $119 \mathrm{NaCl}, 2.5 \mathrm{KCl}$, 6 kynurenic acid, $1 \mathrm{NaH}_{2} \mathrm{PO}_{4}, 13.5$ glucose, 100.1 sucrose, 77.9 $\mathrm{NaHCO}_{3}, 3.5 \mathrm{CaCl}_{2}$ and $\left.7.3 \mathrm{MgCl}_{2}\right)$. Coronal sections $(250 \mu \mathrm{m})$ containing motor cortex, dorsal lateral striatum and dorsal medial striatum were cut using a vibratome (VT1200S; Leica Microsystems, Wetzlar, Germany) in ice-cold cutting solutions. Brain slices were submerged in artificial cerebrospinal fluid (ACSF) (in $\mathrm{mm}$ as follows: $119 \mathrm{NaCl} ; 2.5 \mathrm{KCl}, 1 \mathrm{NaH}_{2} \mathrm{PO}_{4}$, 11 glucose, $26.2 \mathrm{NaHCO}_{3}, 2.5$ $\mathrm{CaCl}_{2}$ and $1.3 \mathrm{MgCl}_{2}$ ) at $27^{\circ} \mathrm{C}$ for $30 \mathrm{~min}$, and equilibrated with $95 \% \mathrm{O}_{2}$ and $5 \% \mathrm{CO}_{2}$. Slices were then individually transferred to the recording chamber and superfused continuously with $32{ }^{\circ} \mathrm{C}$ ACSF containing $50 \mu \mathrm{m}$ picrotoxin.

For the recording of field population spikes, electrodes (3-5 $\mathrm{M} \Omega$ ) were filled with ACSF, and a stimulation intensity of $30 \%$ maximum response is employed. For whole-cell patch-clamp recording, the internal solution contained (in $\mathrm{mM}$ ): $135 \mathrm{KMeSO}_{4}, 10$ $\mathrm{KCl}, 10 \mathrm{HEPES}, 10 \mathrm{Na} 2$-phosphocreatine, $4 \mathrm{MgATP}$, and $0.3 \mathrm{Na}_{3} \mathrm{GTP}$ (pH 7.2-7.4); or $\mathrm{CsMeSO}_{4}$ 130, $\mathrm{NaCl} 10, \mathrm{EGTA} 10, \mathrm{MgATP} 4, \mathrm{Na}_{3} \mathrm{GTP}$ 0.3 and HEPES $10(\mathrm{pH} 7.2-7.4)$. For AMPA/NMDA ratio calculation, the AMPA receptor response was recorded at $-70 \mathrm{mV}$ to prevent NMDA receptor activation, and the NMDA receptor response was estimated at $50 \mathrm{~ms}$ after the peak of mixed currents at $+40 \mathrm{mV}$.
All recordings were filtered $(4 \mathrm{kHz}$ low-pass filter) and sampled $(10 \mathrm{kHz})$ for online and later offline analysis with Multiclamp 700B amplifier (Molecular Devices, Sunnyvale, CA, USA) and Clampfit 10.5 Software.

\section{Human TMS procedures}

Intermittent TMS or continuous theta burst stimulation (cTBS) stimulation were performed as described in previous studies. ${ }^{18,19}$ Briefly, high-frequency intermittent TMS $(10 \mathrm{~Hz}$, strength at $100 \%$ resting motor threshold, $5 \mathrm{~s}$ on and $10 \mathrm{~s}$ off for $10 \mathrm{~min} ; 2000$ pulses) or CTBS $(50 \mathrm{~Hz}$ of $80 \%$ MT for 3 pulses train was repeated at $200 \mathrm{~ms}$ for $40 \mathrm{~s}, 600$ pulses) were applied over the left primary motor cortex with a CCY-I TMS instrument (Yiruide, Wuhan, China) and an ' 8 '-shaped coil for accurately targeted stimulation.

\section{Human MEP procedures}

The MEPs were recorded from abductor pollicis brevis muscle on right hand using the CCY-I TMS affiliated MEP system and analyzed with the CCY-I TMS software. For MEP recording, 20 consecutive MEPs ( $5 \mathrm{~s}$ interval) at each time point were evoked by single pulse TMS stimulation at M1 region, and the peak values were averaged for each time point. Two points of baseline values were taken (separated by $5 \mathrm{~min}$ ) and the plasticity protocol will be applied if there were no more than $10 \%$ differences between the two baseline values. After the plasticity protocol, MEPs were measured after $5,10,15$ and 30 min, respectively. ${ }^{20}$

\section{Rotary rod task for rat}

A rotary rod instrument (Shanghai Xinruan, Shanghai, China) was used in present study. Before the formal test, the animals were briefly familiarized with the rotating rod. The rotating speed was set for each animal to reach an initial latency to fall at $\sim 20 \mathrm{~s}$. Then, the animals were tested for four times with a 10 min interval between tests.

\section{Rotary pursuit task for human subjects}

In rotary pursuit motor learning task, the human subjects were asked to trace a point on the rotating plate with a probe (instrument from Bei Da Qing Niao, Beijing, China), and the time for the probe to successfully contact the rotating point was measured in 4 trials separated by 10 min each. ${ }^{21}$ Before the test, the subjects were familiarized with the test but were allowed less than three trials to prevent overlearning. The speed of the rotating plate was adjusted to reach a starting baseline between 10 and $25 \mathrm{~s}$.

\section{Western blot assays of synaptosomal proteins}

The crude synaptosomal membrane fraction from the motor cortex and dorsal striatum region was purified as described previously. 22 Briefly, brains were removed acutely, and the regions of interests were homogenized by sonication in ice-cold homogenization buffer $(0.32 \mathrm{~m}$ sucrose, $1 \mathrm{~mm}$ EGTA, $1 \mathrm{~mm}$ EDTA, $4 \mathrm{~mm}$ HEPES, pH 7.4) and a protease inhibitor cocktail at $1000 \mathrm{~g}$ at $4{ }^{\circ} \mathrm{C}$ for 10 min for pellet (P1, containing nuclei and large debris) preparation. Then the supernatant $\$ 1$ was centrifuged at $10000 \mathrm{~g}$ at $4{ }^{\circ} \mathrm{C}$ for $30 \mathrm{~min}$ for synaptosomal fraction (P2) and supernatant (S2). The crude synaptosomal membrane pellet (P2) was lysed

Figure 1. Impaired cortical-striatal plasticity and motor skill learning in self-administrated (SA) rats. (a) Nose-poke data of methamphetamine (METH) SA rats $(n=12)$. (b) Motor cortical-cortical long-term potentiation (LTP)-like plasticity was impaired in SA rats ( $n=5$ for control, $n=9$ for METH, $P<0.05$ ). (c) Motor cortical-cortical long-term depression (LTD)-like plasticity was impaired in SA rats $(n=6$ for control, $n=10$ for METH, $P<0.05)$. (d) Motor-dorsal lateral striatum plasticity was impaired in SA rats $(n=5$ for control, $n=6$ for METH, $P<0.05)$. (e) Motor-dorsal medial striatum plasticity remains intact in SA rats $(n=7$ for the control group, $n=9$ for METH, $P>0.05)$. Scale bar for all field excitatory postsynaptic potential (fEPSP) example traces in (c-f): $0.2 \mathrm{mV}, 50 \mathrm{~ms}$. From left to right: recoding site, example trace (gray for after the protocol), group data. Black filled circle: control group; red filled circle: METH group. (f) Motor skill learning was impaired in METH SA rats. Motor skill learning curve was constructed by measuring the latency to fall from the rotating rod $\left(n=10 ; \mathrm{METH}\right.$ vs control, $P<0.01,{ }^{*} P<0.05$ and $\left.{ }^{* *} P<0.01\right)$. 
a

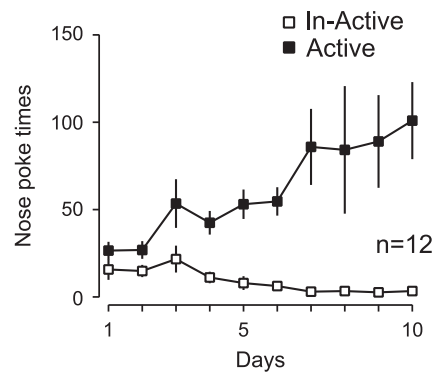

b
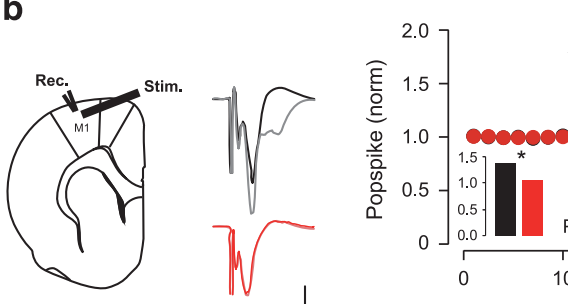

- Control $(n=5)$

- METH $(n=9)$
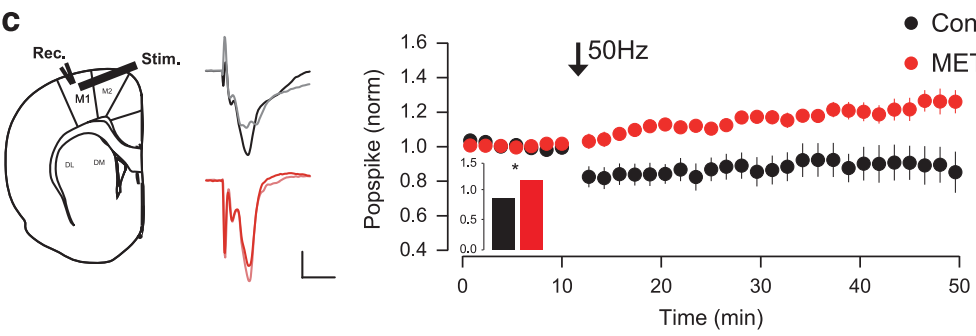

d

- Control $(n=5)$

- METH $(n=6)$
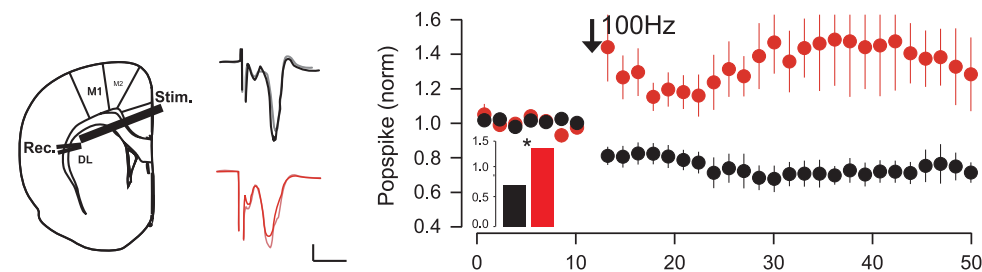

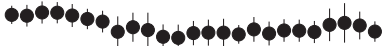

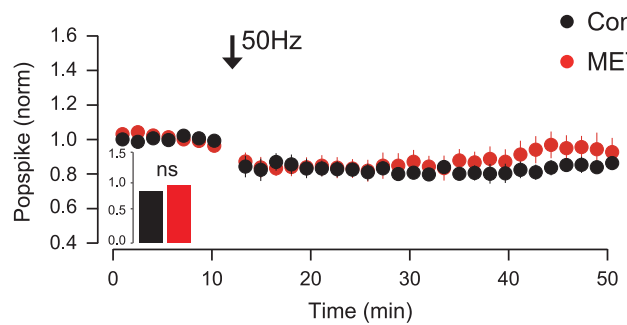

- Control $(n=7)$

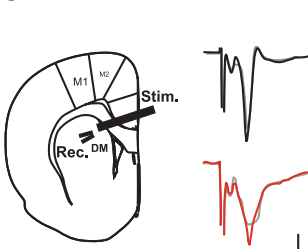

$\operatorname{METH}(n=9)$

f

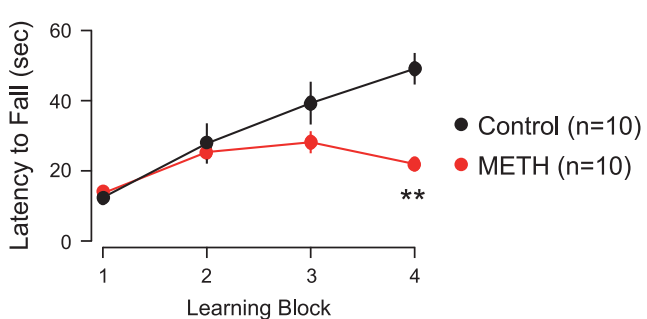

Figure 1. For caption see page on 1275. 
a $\mathrm{MC}$

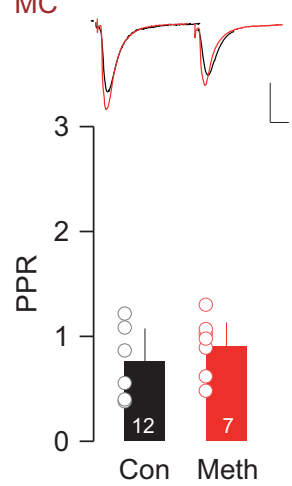

d

MC
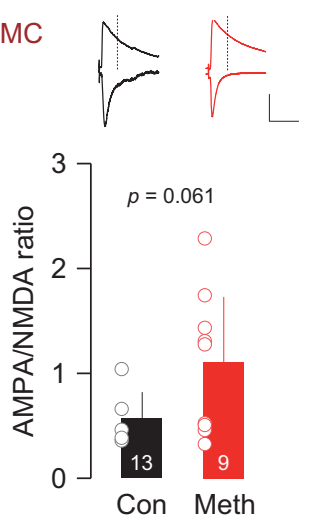

g $\mathrm{DL}$
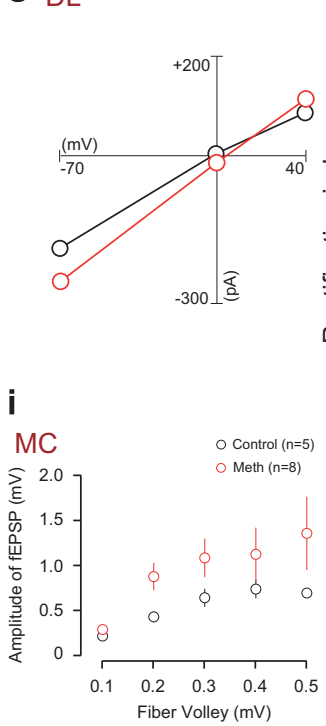

b DL

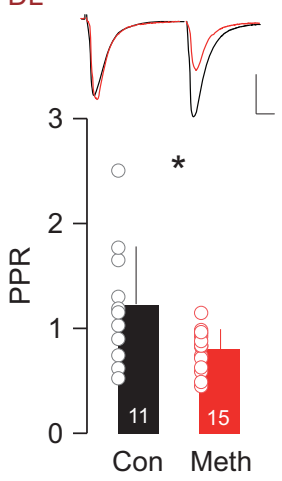

e

DL
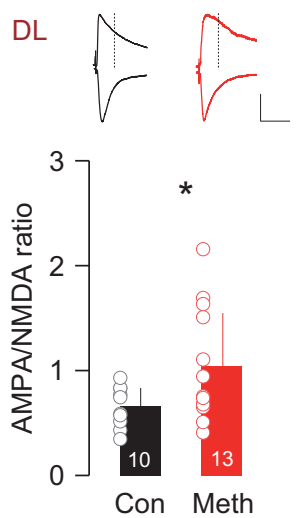

h DM c $\mathrm{DM}$

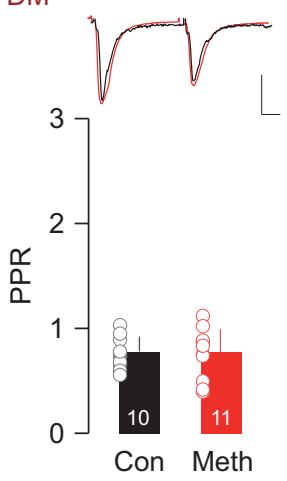

f
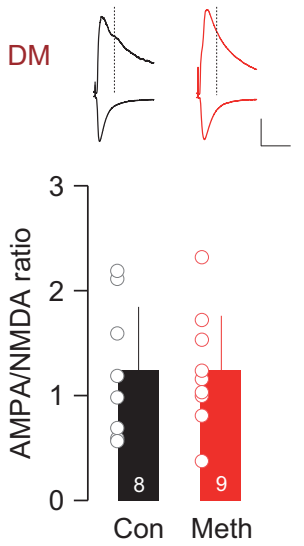

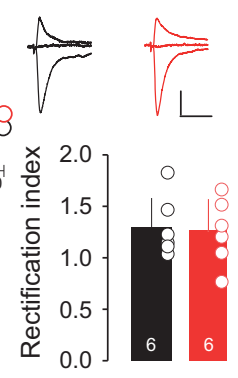

j

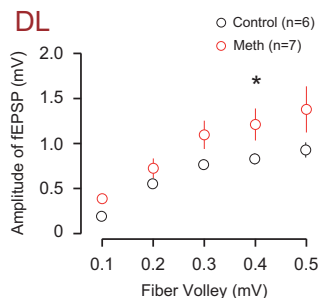

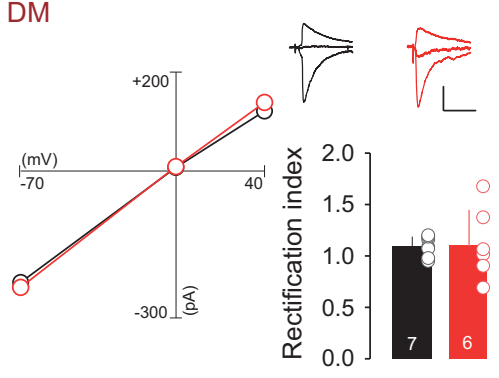

k

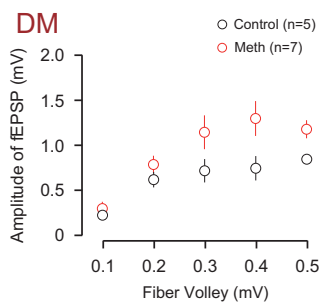

Figure 2. Altered functioning in cortical-striatal synapses. Synaptic transmission parameters (paired-pulse ratio (PPR) (a-c), AMPA/NMDA (A/N) ratio (d-f), retification index (RI) ( $(\mathbf{g}-\mathbf{h})$, input -output curve (i-k)) for Motor cortex (MC), dorsolateral (DL) and dorsomedial (DM) pathway synapses. ${ }^{*} P<0.05$. There were no significant changes in DM and MC synapses, whereas DL pathway revealed decreased PPR value (enhanced presynaptic release) and increased $\mathrm{A} / \mathrm{N}$ ratio (enhanced postsynaptic functions). Scale bars for (a-c): 100 pA, $10 \mathrm{~ms}$. Scale bars for (d-h): 100 pA, 50 ms. Dashed line in (d-f) indicates the estimated NMDA receptor (NMDAR) peak at +40 mV for AMPA/NMDA ratio calculation. AMPA, $\alpha$-amino-3-hydroxy-5-methyl-4-isoxazolepropionic acid; MC, motor cortex; NMDA, N-methyl-D-aspartate; RI, rectification index.

hypo-osmotically and centrifuged at $25000 \mathrm{~g}$ for at $4{ }^{\circ} \mathrm{C}$ for $30 \mathrm{~min}$ to generate the synaptosomal membrane fraction (LP1) and supernatant (LS1). Then, the synaptosomal membrane fraction was solubilized and denatured in Tris-buffered saline containing $0.2 \%$ SDS $\left(70{ }^{\circ} \mathrm{C}, 10 \mathrm{~min}\right)$ diluted in $1 \%$ Triton $\mathrm{X}-100$ and $5 \%$ glycerol and maintained in protease inhibitor cocktail $(50 \mu \mathrm{m}$ MG132 and $10 \mu \mathrm{M} \beta-\mathrm{lac})$.
The $4 \times$ loading buffer (16\% glycerol, $20 \%$ mercaptoethanol, $2 \%$ SDS and $0.05 \%$ bromophenol blue) was added to each sample (3:1 sample/loading buffer) before boiling for $3 \mathrm{~min}$. After coolling down, the samples were subjected to SDS-polyacrylamide gel electrophoresis $\quad\left(10 \% \quad\right.$ acrylamide/0.27\% $\quad N, N^{\prime}$-methylenebisacryalamide resolving gel) for $\sim 40 \mathrm{~min}$ at $80 \mathrm{~V}$ in stacking gel and $\sim 1 \mathrm{~h}$ at $130 \mathrm{~V}$ in resolving gel. The 
a Motor cortex
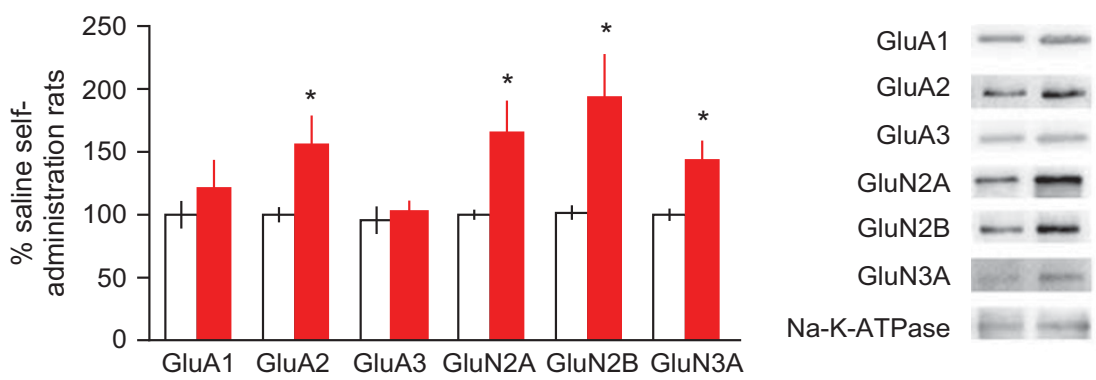

\section{b Dorsal striatum}

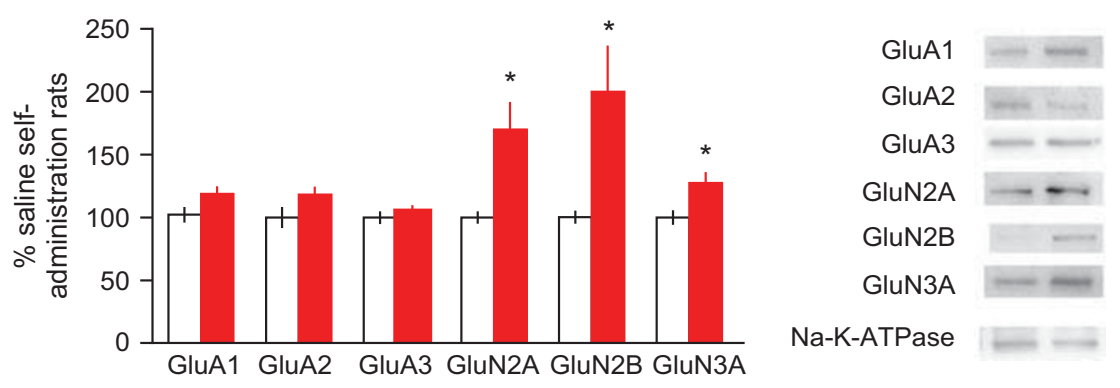

Figure 3. Molecular changes of synaptic glutamate receptors at cortical-striatal synapses. (a) Effects of methamphetamine self-administration (METH SA) on synaptic proteins in the motor cortex. (b) Effects of METH SA on synaptic proteins in dorsal striatum. ${ }^{*} P<0.05$ ( $n=6$; METH vs control).

proteins were electrophoretically transferred onto Immobilon-P transfer membranes (Millipore, Bedford, MA, USA) at $0.25 \mathrm{~A}$ for $2.5 \mathrm{~h}$.

Primary antibodies included anti-GluA1 antibody (1:1000; Abcam, Cambridge, MA, USA, ab109450), anti-GluA2 antibody (1:1000; Abcam; ab52932), anti-GluA3 antibody (1:1000; Abcam, ab40845), anti-GluN2A antibody (1:1000; CST, 4205), anti-GluN2B antibody (1:1000; CST, 4207), anti-GluN3A antibody (1:1000; Santa Cruz, Dallas, TX, USA, sc-51160) or anti-Na-K ATPase antibody (1:1000; Abcam, ab7671). Following overnight incubation at $4{ }^{\circ} \mathrm{C}$, the membranes were incubated for $45 \mathrm{~min}$ at room temperature on a shaker with horseradish peroxidase-conjugated secondary antibody (goat anti-rabbit IgG, ZSGB-BIO, ZB-2301; goat antimouse IgG, ZSGB-BIO, ZB-2305) diluted 1:5000 in blocking buffer. The blots were then screened using the chemiDoc MP system (Bio-Rad, Hercules, CA, USA) for 5-60 s. The level of protein expression was normalized to Na-K ATPase and analyzed with Quantity One software (version 4.4.0; Bio-Rad).

\section{Statistics}

Mean and s.e.m. represented data. Intergroup differences were compared by using independent sample $t$-test with Origin software (Northampton, MA, USA). $P<0.05$ was considered as statistically significant.

\section{RESULTS}

Impaired cortical-striatal plasticity and motor learning after METH $\mathrm{SA}$ in rats

Rats were trained to SA METH for 10 days (Figure 1a).

After 3-9 days of withdrawal, we recorded field potentials in slices of the motor cortex (M1) and the dorsal medial striatum evoked by electrically stimulation as described in a previous study. ${ }^{23}$ We used TBS combined with transient $G_{A B A_{A}}$ receptor blockade to induce long-term potentiation and $50 \mathrm{~Hz}$ stimulation to elicit long-term depression in motor cortex; ${ }^{24}$ we used 100 and $50 \mathrm{~Hz}$ stimulation to induce long-term depression in dorsolateral
(DL) striatum and dorsomedial striatum, ${ }^{23}$ respectively. We found that these stimlation protocols failed to induce synaptic plasticity in METH SA group (Figures $1 \mathrm{~b}-\mathrm{d}$ ) in both the motor cortex and DL striatum, whereas there was no difference in the dorsomedial striatum. This points to a METH effect in the $\mathrm{DL}$, the preferential target of M1 in habit-related drug seeking. ${ }^{25}$ Given the established role of DL striatum in motor learning, we tested the rats for motor skills on a rotarod task. We found that the learning curve of animals in the METH SA group plateaued after the second block (Figure 1f).

Altered synaptic transmission in cortical-striatal synapses To investigate the expression mechanims of METH evoked synaptic plasticity, we established the input-output function, AMPA/NMDA ratio, and the paired-pulse ratio. We found no change in these parameters in the motor cortex M1 (Figures 2a, d and i). In the striatum, neurons of the DL (Figures $2 \mathrm{~b}, \mathrm{e}$ and $\mathrm{j}$ ) but not of the dorsomedial striatum (Figures 2c, $\mathrm{f}$ and $\mathrm{k}$ ) showed a decreased pairedpulse ratio, an increased AMPA/NMDA ratio and steeper input-output function, reflecting enhanced synaptic transmission via increased presynaptic release and upregulated postsynaptic sensitivity.

To examine the subunit composition of AMPA and NMDA receptors, we performed western blot analyses of synaptosomal proteins on both motor cortex and dorsal striatum. Interestingly, we detected increased amount of GluN2A/2B/3A expression in both the motor cortex and the dorsal striatum, suggesting the insertion of the heterotrimeric GluN3A-containing (calcium-impermeable) NMDA receptors (Figures $3 a$ and b). On the other hand, GluA2 protein levels were unaltered, in line with the linear $I-V$ curve after drug exposure, indicating the presence of GluA2-containing AMPARs (Figures $2 \mathrm{~g}$ and $\mathrm{h}$ ).

Diminished motor cortical plasticity and impaired motor learning in human addicts

Next, we examined the impact of chronic METH use on motor cortical plasticity and motor skill learning in human addicts. We 
a

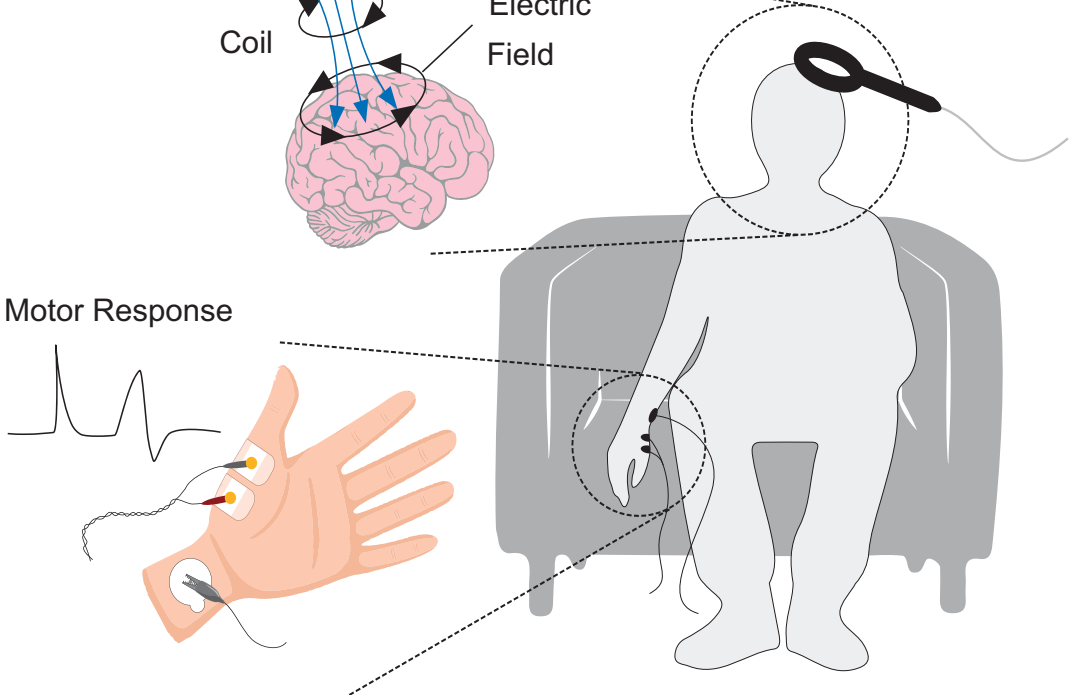

b $\quad 10 \mathrm{~Hz}$ iTMS (induces potentiation)

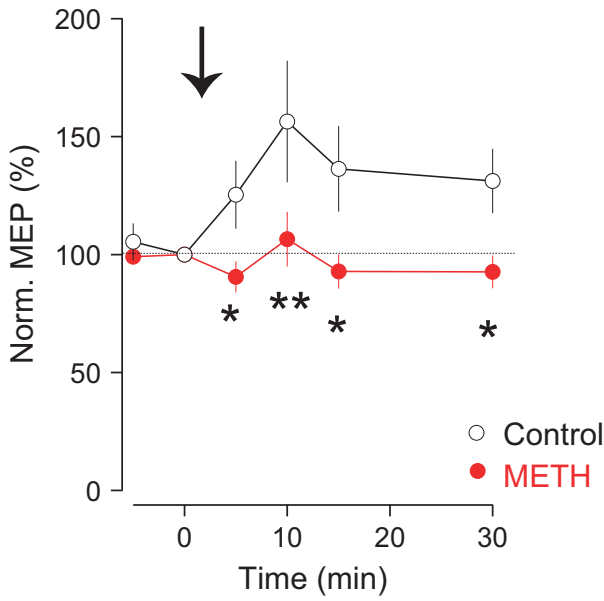

d

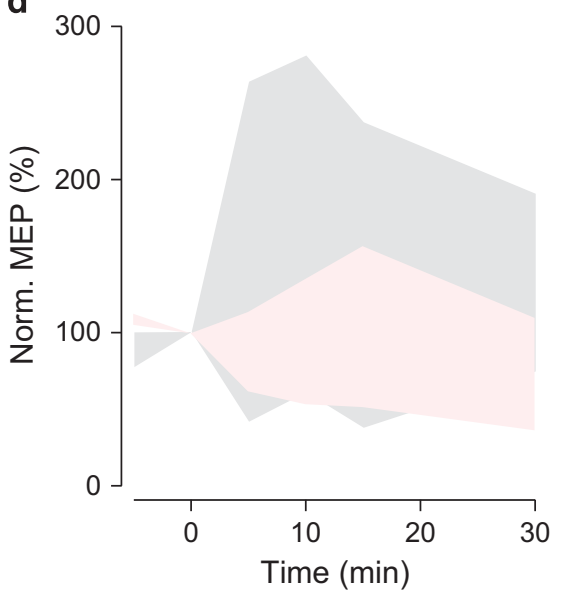

C $\quad$ CTBS (induces depression)

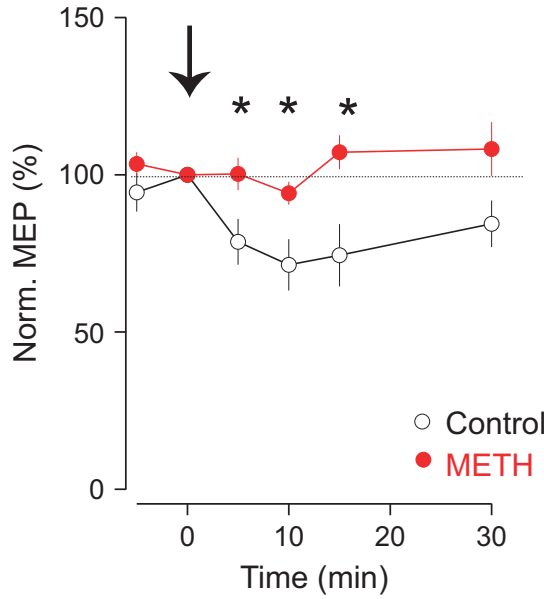

e

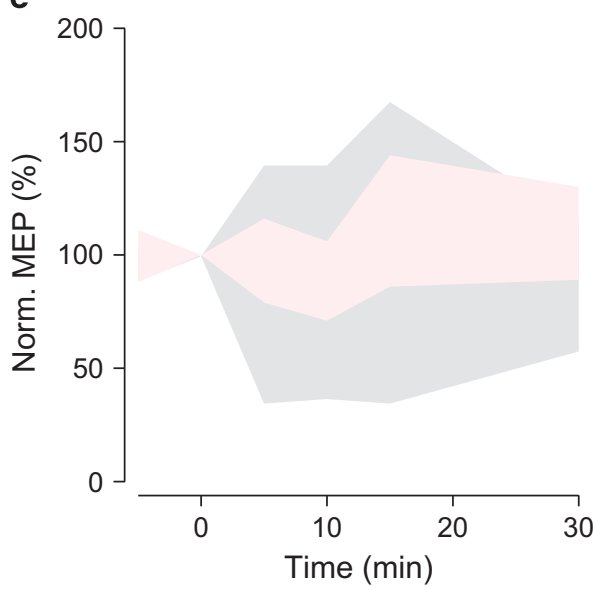

Figure 4. Diminished cortical plasticity in addicts. (a) The design of transcranial magnetic stimulation (TMS) and motor evoked potential (MEP) recordings. Single TMS pulse was administrated on the left motor cortex, and the MEP signal was recorded from abductor pollicis brevis (APB) muscle on right hand. Modified from Shen et al. ${ }^{20}$ with permission. (b) The $10 \mathrm{~Hz}$ repetitive TMS (rTMS) stimulation protocol results in potentiation of MEPs in control but not methamphetamine (METH) group ( $n=11$ for control and $n=18$ for METH); (c) continuous theta burst stimulation (cTBS) protocol results in depression of MEPS in control but not METH group ( $n=9$ for control and $n=11$ for METH); (d, e) control group MEPs exhibit more variance after $10 \mathrm{~Hz}$ cTBS protocol. Black open circle: control group; red filled circle: METH group. ${ }^{*} P<0.05$. 
a Rotary Pursuit Task
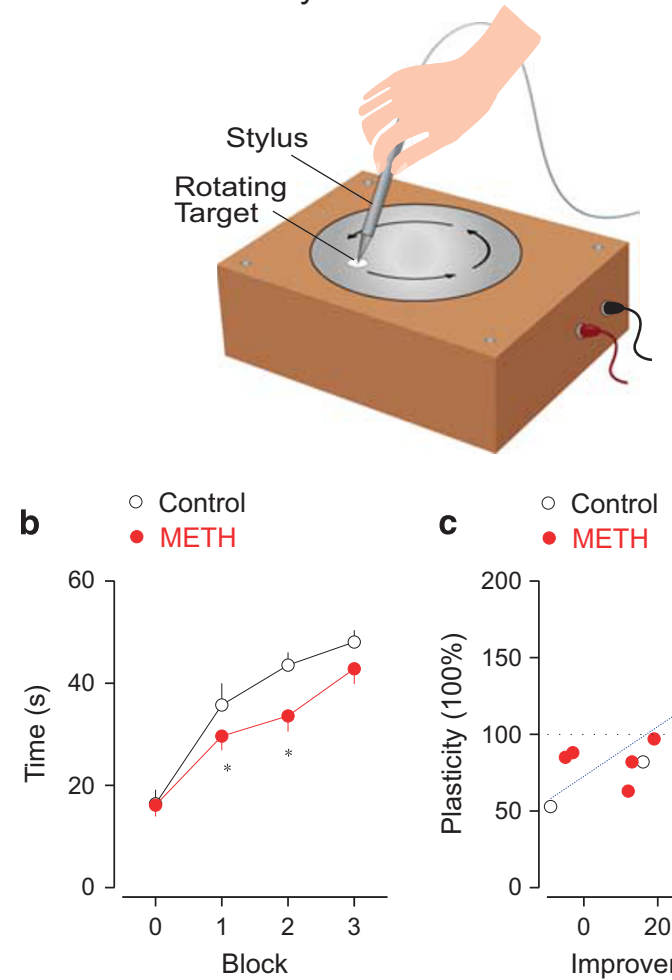

Figure 5. Impaired motor learning correlates with the decreased plasticity. (a) In rotary pursuit motor learning task, the subjects were asked to trace a point on the rotating plate with a probe. Each block lasts for $60 \mathrm{~s}$ (interval at $10 \mathrm{~min}$ ) and the success of time with probe targeting the point was recorded. Modified from Zhou et al. ${ }^{21}$ with permission. (b) The methamphetamine (METH) group exhibits worse performance during rotary pursuit learning task $(n=7$ for control and $n=15$ for METH), $P<0.05$ for second and third tests. (c) The motor learning performance at block two was correlated to the plasticity induction changes at the same time point (Pearson's $r=0.705, P=0.004)$. Black open circle: control group; red filled circle: METH group.

used distinct TMS protocols to potentiate $(10 \mathrm{~Hz}, 10 \mathrm{~min})$ or depress (cTBS, $48 \mathrm{~s}$ ) MEPs (Figure 4a). In the control group, $10 \mathrm{~Hz}$ repetitive TMS stimulation aimed at the motor cortex potentiated MEPs for at least $30 \mathrm{~min}$. In constrast, this potentiation was not obsereved in the group of METH addicts (METH group) (Figure 4b). Similarly, cTBS protocol failed to induce a depression of MEPs in METH group that was robustly observed in the control group (Figure 4c). Notably, with both stimulation protocols, the control group exhibited more individual variability compared with the METH group (Figures $4 d$ and e). Last but not least, the craving score in the METH addict, measured by subjective responses to a craving scale, inversely correlated with the MEP plasticity at 10 min time point (Pearson's $r=-0.6315$; adjusted $r^{2}=0.30$ ).

Finally, we tested the motor skill learning ability in addicts with a rotary pursuit learning task (Figure $5 \mathrm{a}$ ). The subjects in the METH group took more time to learn the task compared with the control group (Figure 5b). Notably, the motor learning performance correlated with the plasticity of the MEP elicited by $10 \mathrm{~Hz}$ stimulation protocol (Figure $5 \mathrm{c}$ ).

\section{DISCUSSION}

We report that METH abuse can affect plasticity of the motor cortex in both drug-exposed rats and human addicts. The reduced cortical plasticity in addicts argues for an involvement of motor system in advanced stages of the disease, in line with the hypothesis that with the progression of addiction, the dorsal striatum becomes involved. ${ }^{8,25,26}$ Moreover, the poor learning ability could reflect a reduced flexibility that contributes to maintenance of the compulsive behavior and inhibits recovery.

One potential molecular correlate of a reduced synaptic plasticity is the insertion of calcium-impermeable (GluN3Acontaining) NMDA receptors that were detected on midbrain DA neuron at an early stage of drug exposure, ${ }^{3}$ as well as in striatal neurons in degenerative diseases. ${ }^{27}$ NMDA receptors in the cortex are also essential for many forms of plasticity, and the presence of calcium-impermeable NMDA receptors alter the rules for plasticity induction. ${ }^{28}$ Here we confirm the insertion of GluN3A by western blot, supporting the notion that a failed induction mechanism underlies the impaired bidirectional plasticity.

Another mechanism underlying the diminished cortical plasticity may be the loss of dopaminergic signaling. ${ }^{29}$ For instance, human METH addicts exhibit decreased DA transmission in the brain that may manifest as drug-induced parkinsonism. ${ }^{30-33}$ In the present study we found a delayed motor learning ability in addicted patients. Future studies will be required to understand whether other types of motor learning than the pursuit task tested here are also impaired after drug abuse.

We also provide evidence for a decreased intrinsic excitability of neurons in cortical-striatal circuits after repeated METH exposure. ${ }^{2,34}$ The reduced response to TMS protocols could be partly explained by the failure to reach the firing threshold in neurons that are less excitable.

One limitation of the present study is that with electrical stimulation, the exact synaptic pathways in the motor-striatal circuit cannot be reliably identified. Employing optogenetic tools that allow further dissection will be helpful to identify the circuits affected by these changes. On the human study side, the TMSinduced cortical plasticity can be explained by either synaptic plasticity or changes of intrinsic excitability. To refine stimulation protocols that could restore normal plasticity, it will be important to examine which of the two potential mechanisms applies.

In summary, the present study demonstrates an unexpected change in motor cortical plasticity after METH abuse. Clinical studies are needed to explore the relationships between altered motor cortical plasticity and addiction symptoms (for example, craving, relapse). This will enable translation to the clinic of rational therapy for drug addiction, a disease with a tremendous burden for society.

\section{CONFLICT OF INTEREST}

The authors declare no conflict of interest.

\section{ACKNOWLEDGMENTS}

We thank Meaghan Creed, Christian Luscher and Burton Slotnick for help in preparation of the manuscript. The study is supported by NSFC Grant (81501164, 81611130224) and Jiangsu Provincial NSF (BK20140917) to T-FY. T-FY is also supported by Young Elite Scientists Sponsorship Program by CAST (YESS). This study was supported by Macao Science and Technology Development Fund (063/2015/A2) and multi-year research grant, University of Macau, MYRG2016-00184-ICMS-QRCM to H.S.; O.A-C was supported by CONACYT-FOSISS 2016 (Grant 273213); Y-X.X is supported by National Basic Research Program of China (2015CB559200) and Beijing Natural Science Foundation (No. 5162015) and Ten-Thousand Youth Talents Projects.

\section{AUTHOR CONTRIBUTIONS}

$X H, Y-Y C, O A-C, Y-X X, H S$ and T-FY designed the study; $X H, Y-Y C, Y S, X C, A L, Q L$, $Z \mathrm{~L}, \mathrm{~L}-\mathrm{BZ}$ and WD performed the study (XH for animal electrophysiology, $\mathrm{Y}-\mathrm{YC}$, $\mathrm{ZL}$ and $\mathrm{L}-\mathrm{BZ}$ for self-administration training and western blot, $\mathrm{YS}, \mathrm{XC}, \mathrm{QL}$ and WD for human TMS and MEP experiment and AL for animal behavior); $\mathrm{XH}, \mathrm{Y}-\mathrm{YC}$, $\mathrm{OA}-\mathrm{C}, \mathrm{Y}-\mathrm{XX}, \mathrm{HS}$ and T-FY analyzed the results and wrote the paper together; all authors have read and approved the final version of the manuscript. 


\section{REFERENCES}

1 Pignatelli $M$, Bonci $A$. Role of dopamine neurons in reward and aversion: a synaptic plasticity perspective. Neuron 2015; 86: 1145-1157.

2 Kourrich S, Calu DJ, Bonci A. Intrinsic plasticity: an emerging player in addiction. Nat Rev Neurosci 2015; 16: 173-184.

3 Yuan T, Mameli M, O'Connor EC, Dey PN, Verpelli C, Sala C et al. Expression of cocaine-evoked synaptic plasticity by GluN3A-containing NMDA receptors. Neuron 2013; 80: 1025-1038.

4 Ungless MA, Whistler JL, Malenka RC, Bonci A. Single cocaine exposure in vivo induces long-term potentiation in dopamine neurons. Nature 2001; 411: 583-587.

5 Conrad KL, Tseng KY, Uejima JL, Reimers JM, Heng LJ, Shaham Y et al. Formation of accumbens GluR2-lacking AMPA receptors mediates incubation of cocaine craving. Nature 2008; 454: 118-121.

6 Luscher C, Malenka RC. Drug-evoked synaptic plasticity in addiction: from molecular changes to circuit remodeling. Neuron 2011; 69: 650-663.

7 Goldstein RZ, Volkow ND. Dysfunction of the prefrontal cortex in addiction: neuroimaging findings and clinical implications. Nat Rev Neurosci 2011; 12: 652-669.

8 Everitt BJ, Robbins TW. Neural systems of reinforcement for drug addiction: from actions to habits to compulsion. Nat Neurosci 2005; 8: 1481-1489.

9 Yalachkov Y, Kaiser J, Naumer MJ. Sensory and motor aspects of addiction. Behav Brain Res 2010; 207: 215-222.

10 Kosten TR, Scanley BE, Tucker KA, Oliveto A, Prince C, Sinha R et al. Cue-induced brain activity changes and relapse in cocaine-dependent patients. Neuropsychopharmacology 2006; 31: 644-650.

11 Smolka MN, Buhler M, Klein S, Zimmermann U, Mann K, Heinz A et al. Severity of nicotine dependence modulates cue-induced brain activity in regions involved in motor preparation and imagery. Psychopharmacology (Berl) 2006; 184: 577-588.

12 Yalachkov Y, Kaiser J, Naumer MJ. Brain regions related to tool use and action knowledge reflect nicotine dependence. J Neurosci 2009; 29: 4922-4929.

13 Brody AL, Mandelkern MA, Olmstead RE, Jou J, Tiongson E, Allen V et al. Neural substrates of resisting craving during cigarette cue exposure. Biol Psychiatry 2007; 62: 642-651.

14 London ED, Kohno M, Morales AM, Ballard ME. Chronic methamphetamine abuse and corticostriatal deficits revealed by neuroimaging. Brain Res 2015; 1628(Pt A): 174-185.

15 Willuhn I, Steiner H. Motor-skill learning-associated gene regulation in the striatum: effects of cocaine. Neuropsychopharmacology 2006; 31: 2669-2682.

16 Xue YX, Luo YX, Wu P, Shi HS, Xue LF, Chen C et al. A memory retrieval-extinction procedure to prevent drug craving and relapse. Science 2012; 336: 241-245.

17 Luo YX, Xue YX, Liu JF, Shi HS, Jian M, Han Y et al. A novel UCS memory retrievalextinction procedure to inhibit relapse to drug seeking. Nat Commun 2015; 6: 7675.

18 Huang YZ, Edwards MJ, Rounis E, Bhatia KP, Rothwell JC. Theta burst stimulation of the human motor cortex. Neuron 2005; 45: 201-206.

19 Shen Y, Cao X, Tan T, Shan C, Wang Y, Pan J et al. 10-Hz repetitive transcranial magnetic stimulation of the left dorsolateral prefrontal cortex reduces heroin cue craving in long-term addicts. Biol Psychiatry 2016; 80: e13-e14.

20 Shen Y, Cao X, Shan C, Dai W, Yuan TF. Heroin addiction impairs human cortical plasticity. Biol Psychiatry 2017; 81: e49-e50.

21 Zhou D, Pang F, Liu S, Shen Y, Liu L, Fang Z et al. Altered motor-striatal plasticity and cortical functioning in patients with schizophrenia. Neurosci Bull 2017; 33: 307-311.
22 Ren ZY, Liu MM, Xue YX, Ding ZB, Xue LF, Zhai SD et al. A critical role for protein degradation in the nucleus accumbens core in cocaine reward memory. Neuropsychopharmacology 2013; 38: 778-790.

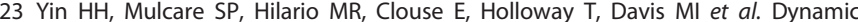
reorganization of striatal circuits during the acquisition and consolidation of a skill. Nat Neurosci 2009; 12: 333-341.

24 Harms KJ, Rioult-Pedotti MS, Carter DR, Dunaevsky A. Transient spine expansion and learning-induced plasticity in layer 1 primary motor cortex. J Neurosci 2008; 28: $5686-5690$.

25 Everitt BJ, Robbins TW. From the ventral to the dorsal striatum: devolving views of their roles in drug addiction. Neurosci Biobehav Rev 2013; 37(9 Pt A): 1946-1954.

26 Luscher C, Bellone C. Cocaine-evoked synaptic plasticity: a key to addiction? Nat Neurosci 2008; 11: 737-738.

27 Marco S, Giralt A, Petrovic MM, Pouladi MA, Martinez-Turrillas R, Martinez-Hernandez $\mathrm{J}$ et al. Suppressing aberrant GluN3A expression rescues synaptic and behavioral impairments in Huntington's disease models. Nat Med 2013; 19: 1030-1038.

28 Henson MA, Roberts AC, Perez-Otano I, Philpot BD. Influence of the NR3A subunit on NMDA receptor functions. Prog Neurobiol 2010; 91: 23-37.

29 Molina-Luna K, Pekanovic A, Rohrich S, Hertler B, Schubring-Giese M, RioultPedotti MS et al. Dopamine in motor cortex is necessary for skill learning and synaptic plasticity. PLOS ONE 2009; 4: e7082.

30 Zorick T, Lee B, Mandelkern MA, Fong T, Robertson C, Ghahremani DG et al. Low striatal dopamine receptor availability linked to caloric intake during abstinence from chronic methamphetamine abuse. Mol Psychiatry 2012; 17: 569-571.

31 Volkow ND, Chang L, Wang GJ, Fowler JS, Leonido-Yee M, Franceschi D et al. Association of dopamine transporter reduction with psychomotor impairment in methamphetamine abusers. Am J Psychiatry 2001; 158: 377-382.

32 Wang GJ, Smith L, Volkow ND, Telang F, Logan J, Tomasi D et al. Decreased dopamine activity predicts relapse in methamphetamine abusers. Mol Psychiatry 2012; 17: 918-925.

33 Krasnova IN, Justinova Z, Ladenheim B, Jayanthi S, McCoy MT, Barnes C et al. Methamphetamine self-administration is associated with persistent biochemical alterations in striatal and cortical dopaminergic terminals in the rat. PLOS ONE 2010; 5: e8790.

34 Chen BT, Yau HJ, Hatch C, Kusumoto-Yoshida I, Cho SL, Hopf FW et al. Rescuing cocaine-induced prefrontal cortex hypoactivity prevents compulsive cocaine seeking. Nature 2013; 496: 359-362.

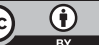

This work is licensed under a Creative Commons Attribution 4.0 International License. The images or other third party material in this article are included in the article's Creative Commons license, unless indicated otherwise in the credit line; if the material is not included under the Creative Commons license, users will need to obtain permission from the license holder to reproduce the material. To view a copy of this license, visit http://creativecommons.org/licenses/ by/4.0/

(c) The Author(s) 2017 